\title{
Improved accuracy of short-distance measurement of water flow velocity using Pulse Boundary Model
}

\author{
Yuequn Dong ${ }^{1,2, *}$, Tingwu Lei ${ }^{3,4}$, Qingwen Zhang ${ }^{1}$, Xiaohui Zhuang ${ }^{3}$, and Fangfang Liu ${ }^{3}$ \\ ${ }^{1}$ Agricultural Clear Watershed Group, Institute of Agro-environment and Sustainable Development, CAAS, Beijing, China \\ ${ }^{2}$ Beijing Enterprises Water Group Limited, Beijing, China \\ ${ }^{3}$ College of Water Resources and Civil Engineering, China Agricultural University, Beijing, China \\ ${ }^{4}$ State Key Laboratory of Soil Erosion and Dryland Farming on the Loess Plateau, Institute of Soil and Water Conservation, CAS \\ and MWR, Yangling, China
}

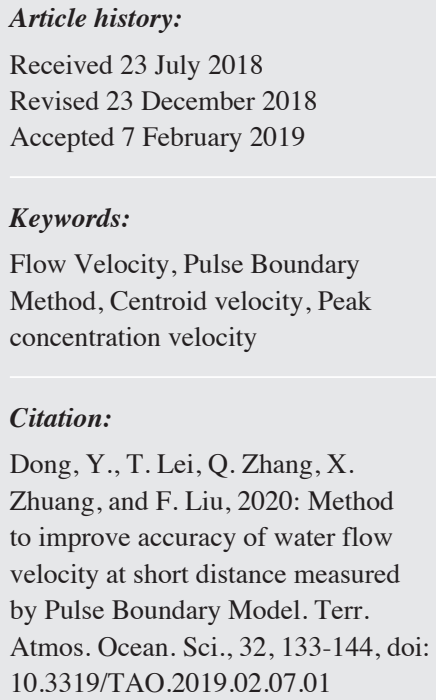

\begin{abstract}
Salt tracer is one of the widely used shallow water velocity measurement methods. The Pulse Boundary Model method produces low velocity at short distances from the salt injection position. This study proposes a two-step approach to accurately estimate the flow velocity. Experiments were carried out under three flow rates of 12,24 , and $48 \mathrm{~L} \mathrm{~min}^{-1}$ and three slope gradients of 4,8 , and $12^{\circ}$ at six measurement positions of $0.05,0.3,0.6,0.9,1.2$, and $1.5 \mathrm{~m}$ from the solute injection positions. The new method obtains peak velocities that are 0.999 times those of the centroid velocities, indicating that either centroid or peak time can be equally used to measure flow velocity. The new method significantly improves measurement accuracy of flow velocity at short distances, as indicated by the almost equal measured velocities at all locations as those measured at longer distances. Velocities measured by the new method were significantly higher than those measured by the Pulse Boundary Model method or the centroid velocities measured by the traditional salt tracer method. In addition, the centroid and the peak velocities obtained by the new method correlates well to those by the traditional volumetric method. The velocities measured by the volumetric method were 0.79 (centroid velocity) and 0.78 (peak velocity) times of those estimated by the new and improved method. The results show that new and improved method provides an accurate and efficient approach in measuring shallow water flow velocity at short distances.
\end{abstract}

\section{INTRODUCTION}

The velocity of overland flow is one of the most important parameters in predicting soil erosion and non-point source pollution. This factor is the key to understand the complicated mechanism of soil erosion and has long been a focus of concern in developing and improving processbased erosion prediction models. Thus, the accurate measurement of shallow water flow velocity is of interest in both laboratory and field conditions.

Water flow along the soil slope commonly has a depth of $\mathrm{mm}$ or $\mathrm{cm}$. The velocity of this shallow water flow is influenced by many factors under specific circumstances. The existing measurement for flow velocity include tracers

\footnotetext{
* Corresponding author

E-mail:dongyuequn@163.com
}

of dye, salt, and thermal (Bresler 1973; Luk and Merz 1992; Lei et al. 2010; Zhang et al. 2010; Abrantes et al.2018), volumetric, hot film, Particle Image Velocimetry (PIV), photoelectric sensor, and conductance sensor methods (Bruun 1996; Li et al. 1997; Hyun et al. 2003; Giménez et al. 2004; Liu et al. 2007, 2008). However, most of these measurements are for the velocity of open-channel flow. Among these different methods, the dye and salt tracers are lowcost and easy to operate while the others are high in cost, not suitable for the measurement of shallow flow velocity, or not environmentally friendly, such as the radioisotope tracer method.

The traditional dye tracer method is widely used to measure velocities of flow water. However, the velocities need to be re-calibrated or corrected with different factors, 
such as correction coefficients from 0.365 to 0.825 (Horton et al. 1934; Emmett 1970; Dunkerley 2001; Myers 2002; Zhang et al. 2010). The calibration parameters are related to the flow velocity, diffusion dispersion coefficient, and the sediment content in the flow (Planchon et al. 2005), and the value of 0.67 was widely used (Rouhipour et al. 1999; Pan and Shangguan 2006). In addition, the dye tracer method needs a relatively long distance for measurement because dye diffusion and systematic error may cause considerable error at very short distances. However, with the development of photology, greater accuracy can be attained by using infrared video (Abrantes et al. 2018).

The salt tracer method has been used to measure shallow water flow velocities for many years. Along with different mathematical models (Lei et al. 2005, 2010; Shi et al. 2012), the traditional salt tracer method can estimate flow velocity by measuring the conductivity using electric sensors (Li et al. 1996; Chang et al. 2015). The distance of salt injection to the tracer sensor is divided by the correlating identified peak detection time, leading edge detection time, or the calculated centroid time from the pulse tracer curve. Thus, not all measured data contribute to the velocity calculation but rather only the time to peak concentration, leading edge, or the centroid of the tracer curve are considered. However, the time of peak concentration or leading edge is difficult to determine when the measured curve quality is poor, which indicates an irregular or missing peak, or unclarity of leading edge due to electronic noise or unsteady water flow.

For the mathematical methods, the Pulse Boundary Model proposed by Lei et al. (2005) is based on the instant injection of electrolyte solution, which is considered as a pulse function. The boundary condition needs to obtain the analytical solution of the one-dimensional convection dispersion equation under steady water flow. By fitting this solution into the experimentally measured solute transport process data, the average velocity can be determined. Results calculated by the Pulse Boundary Model turns out to be lower than the real velocity (Lei et al. 2010) because the solute injection is never actually instantaneous but rather always takes a certain period of time, often longer than $0.6 \mathrm{~s}$. Therefore, the pulse boundary is merely a mathematical approximation of the real velocity. In addition, this error is even higher when the measured distance from the salt injection point is short, which requires a relatively long duration for the salt injection. For instance, the flow velocity of $1 \mathrm{~m} \mathrm{~s}^{-1}$ with a measurement distance shorter than $1 \mathrm{~m}$ from the salt injection point results in a measurement error of approximately $60 \%$. However, the Pulse Boundary Model can present an excellent model fit to the measured data. This indicates that the model simulation data followed the peak position and the rising and falling trends of the resulting curves. Thus, the peak detection time can be effectively detected.
In this study, a combined method for estimating shallow flow velocity is proposed to take advantage of the excellent fit of the Pulse Boundary Model by Lei et al. (2005) and the simple calculation of the traditional salt tracer method. The specific purposes of this study are: (1) to outline the procedures for the combination of the Pulse Boundary Model and traditional salt tracer methods; (2) to estimate the velocity with the experimental data using the new and improved method; (3) to validate the method results by comparing the measured velocities with those obtained by the volumetric method; and (4) to estimate the relative errors of Pulse Boundary Model at different positions under the experimental conditions.

\section{MODEL DEVELOPMENT}

\subsection{Theoretical Background}

\subsubsection{Velocity Estimated by the Traditional Salt Tracer Method}

For a finite travel distance, soil infiltration and adsorption of salt can be ignored. According to the classic mechanical principle, the centroid velocity of salt pulse is consistent with the water flow velocity (Elder 1959). Thus, for the traditional salt tracer method, the flow velocity is estimated by measuring the conductivity, which represents the processes of solute transport. The peak time $t_{p}$, leading edge time $t_{e}$, and the centroid time $t_{c}$ (Fig. 1) of the tracer curve at a sampling station downslope are obtained. The travel distance is then divided by the time to reach the peak velocity $u_{p}$, leading edge velocity $u_{e}$, and the centroid velocity $u_{c} . t_{e}$ and $t_{p}$ can be identified from the tracer curves, while $t_{c}$ involves further computations. Peak velocity, centroid velocity, and centroid time can be calculated using Eqs. (1) to (3).

$$
\begin{aligned}
& u_{p n}=\frac{L_{n}}{t_{p n}} \\
& u_{c n}=\frac{L_{n}}{t_{c n}} \\
& t_{c n}=\frac{\sum_{t_{e}}^{t_{f}} C_{n i} t_{n i}}{\sum_{t_{c}}^{t_{f}} C_{n i}}
\end{aligned}
$$

where $n$ is the sensor sequence number (Fig. 1), $n=1 \sim 6$; $L_{n}$ is the distance from the solute injection position to the $n$th sensor; $t_{p}(\mathrm{~s})$ is the peak detection time of tracer curves; $t_{f}(\mathrm{~s})$ is the ending edge detection time of the pulse tracer curve; $t_{n i}(\mathrm{~s})$ is the time corresponding to a certain data point of the $n$th sensor; and $C_{n i}$ is the conductivity corresponding to a certain data point of the $n$th sensor. 


\subsubsection{Velocity Estimated by the Pulse Boundary Model Method}

Salt solution is transported in water flow through both convection and dispersion mechanisms. Many factors influence this transportation process, such as flow rate, flow velocity, and water quality. A shallow water flow, which is generally in $\mathrm{cm}$, can reasonably be assumed as a steady flow and can be treated as one-dimensional along the stream line. The convectional and dispersion of salt (chemical) in a steady water flow is defined by Fick's law and the mass conservation law, given by the differential equation for the one-dimensional solute transport as:

$\frac{\partial C}{\partial t}+u \frac{\partial C}{\partial x}=\frac{\partial}{\partial x}\left(D_{H} \frac{\partial C}{\partial x}\right)$

where $C$ is the solute concentration, $\mathrm{kg} \cdot \mathrm{m}^{-3}$, a function of distance $x$ and time $t$, proportional to the electrical conductivity of the solution; $x(\mathrm{~m})$ is the coordinate along the slope; $u\left(\mathrm{~m} \cdot \mathrm{s}^{-1}\right)$ is flow velocity; $t(\mathrm{~s})$ is time; and $D_{H}\left(\mathrm{~m}^{2} \cdot \mathrm{s}^{-1}\right)$ is the hydrodynamic dispersion coefficient.

If the upper boundary condition is assumed to be a pulse, then the initial and boundary conditions for Eq. (4) are given as:

$C(x, t)=C_{0} \delta(t) \quad x=0$

$C(x, t)=0 \quad x=\infty$

$C(x, t)=0 \quad t=0$

The solution to Eq. (4) as a time-dependent function is given by Lei et al. (2005) as:

$C(x, t)=C_{0} \frac{x}{2 t \sqrt{\pi D_{H} t}} \exp \left[-\frac{(x-u t)^{2}}{4 D_{H} t}\right]$
The experimentally obtained data are fitted with Eq. (6), based on the least squares method (Lei et al. 2005), to produce the flow velocity, $u$, and the hydrodynamic dispersion coefficient, $D_{H}$.

\subsubsection{Relationship Between the Traditional Salt Tracer and Pulse Boundary Model Methods}

As concluded above, the centroid velocity of the salt pulse is consistent with the water flow velocity. In addition, the velocity derived from the traditional salt tracer and the Pulse Boundary Model methods are consistent, based on the following theoretical analysis (Xia 2003).

Figure 2 shows that $C(x, t)$, a function of distance $x$ and time $t$, is a continuous function. Thus, the computation [Eq. (3)] can be changed to:

$t_{c}=\frac{\int_{0}^{\infty} C(x, t) t d t}{\int_{0}^{\infty} C(x, t) d t}$

Substituting Eqs. (6) into (7) leads to:

$t_{c}=\frac{\int_{0}^{\infty} \frac{1}{\sqrt{t}} \exp \left(\frac{-u^{2} t}{4 D_{H}}-\frac{x^{2}}{4 D_{H} t}\right) d t}{\int_{0}^{\infty} \frac{1}{\sqrt{t^{3}}} \exp \left(\frac{-u^{2} t}{4 D_{H}}-\frac{x^{2}}{4 D_{H} t}\right) d t}$

Let:

$a_{1}=\frac{u^{2}}{4 D_{H}}$

$a_{2}=\frac{x^{2}}{4 D_{H}}$

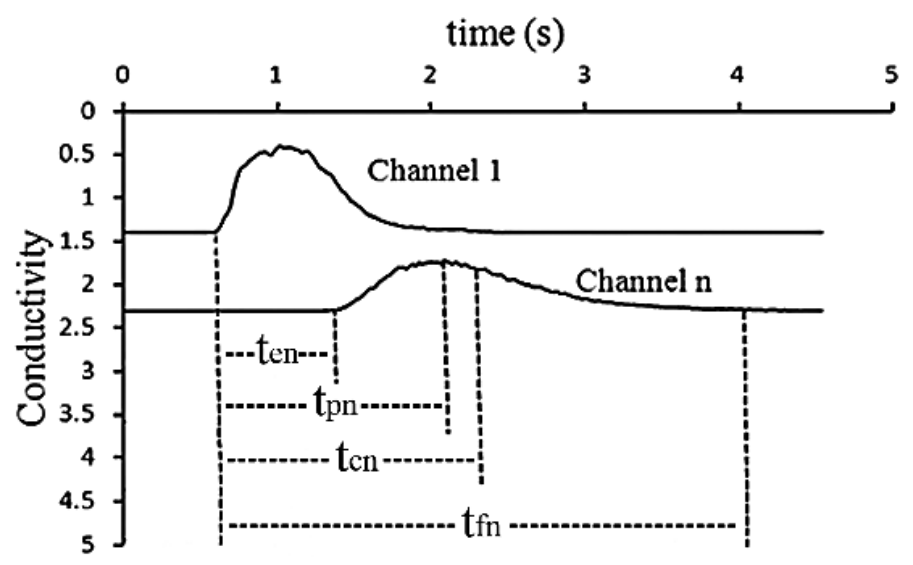

Fig. 1. Temporal distribution of electric conductivity. 

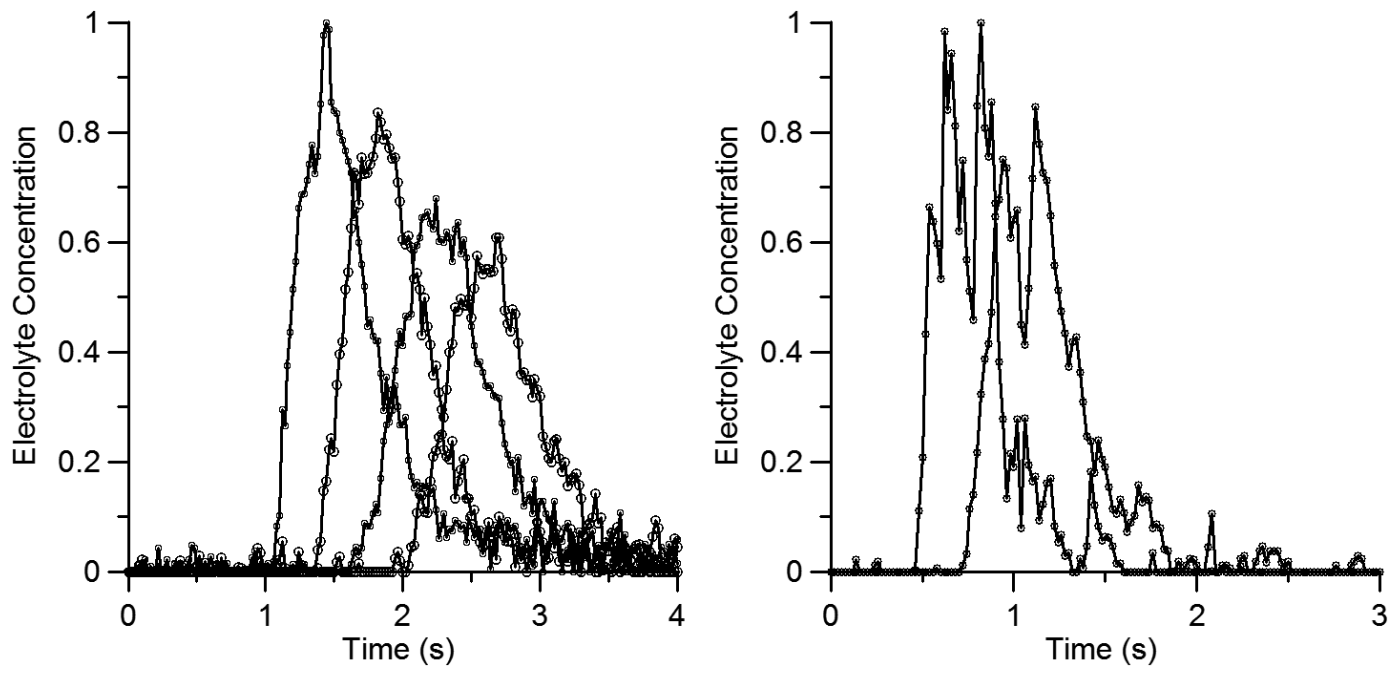

Fig. 2. Tracer curves from actual experiments.

Then we obtain:

$$
t_{c}=\frac{\int_{0}^{\infty} \frac{1}{\sqrt{t}} \exp \left(-a_{1} t-\frac{a_{2}}{t}\right) d t}{\int_{0}^{\infty} \frac{1}{\sqrt{t^{3}}} \exp \left(-a_{1} t-\frac{a_{2}}{t}\right) d t}
$$

The form can be changed to:

$$
t_{c}=\frac{\int_{0}^{\infty} \frac{\exp \left[-\frac{\left(\sqrt{4 a_{2}}\right)^{2}}{4 t}\right] \exp \left(-a_{1} t\right)}{\sqrt{t}} d t}{\int_{0}^{\infty} \frac{\exp \left[-\frac{\left(\sqrt{4 a_{2}}\right)^{2}}{4 t}\right] \exp \left(-a_{1} t\right)}{\sqrt{t^{3}}} d t}
$$

Use the inverse Laplace transformation to determine:

$t_{c}=\frac{\sqrt{\frac{\pi}{a_{1}}} \exp \left(-\sqrt{4 a_{2} s}\right)}{\sqrt{\frac{4 \pi}{4 a_{2}}} \exp \left(-\sqrt{4 a_{2} s}\right)}$

Simplify and obtain:

$t_{c}=\sqrt{\frac{a_{2}}{a_{1}}}$

Substitute Eqs. (9) and (10) into (14) and we can determine:

$t_{c}=\frac{x}{u}$
Equation (15) shows that velocities that derived from the traditional salt tracer and Pulse Boundary Model methods are theoretically consistent (Xia 2003). Thus, we can use the solution of Pulse Boundary Model to calculate the centroid time. On this basis, we can conclude that the coupling of Pulse Boundary Model and salt tracer method is theoretically rational.

\subsection{The Coupled Pulse Boundary Model and Traditional Salt Tracer Method}

Centroid velocity derived from the traditional salt tracer method has a more acceptable physical meaning and is easy to obtain. However, different experimental conditions can cause varying water and laminar flows or salt adsorption by the sediment, which in turn results in erratic measurement curves. Figure 2 shows that the measured tracer curves are jagged rather than smooth lines, and the velocities derived from the calculated centroid times also vary dramatically. As mentioned above, velocities derived from the traditional salt tracer and Pulse Boundary Model methods are theoretically consistent. Thus, we propose a new calculation that combines these two methods. First, fit the experimentally obtained data, electrolyte concentration, and time with the solution of Eq. (6) to obtain the fitted curves of the Pulse Boundary Model, dispersion coefficient $D_{H}$, and the flow velocity $u_{\mathrm{e}}$. The fitted peak time $t_{p}^{\prime}$ was identified from the fitted curve, and the centroid time $t_{c}^{\prime}$ from fitted curves was calculated using Eq. (3). Then, determine the elapsed centroid time between the adjacent sensors of the fitted curve $\Delta t_{c n}^{\prime}$ with Eq. (16), and the peak concentration time elapsed between the adjacent sensors of the fitted curve $\Delta t_{p n}^{\prime}$ with Eq. (18). Finally, calculate the centroid velocity $u_{c n}^{\prime}$ and peak concentration velocity $u_{p n}^{\prime}$ using the new 
and improved method with Eqs. (17) and (18). The relevant equations are:

$$
\begin{aligned}
& \Delta t_{c n}^{\prime}=t_{c(n+1)}^{\prime}-t_{c n}^{\prime} \\
& u_{c n}^{\prime}=\frac{L_{n}}{\Delta t_{c n}^{\prime}} \quad(n=1 \sim 5) \\
& \Delta t_{p n}^{\prime}=t_{p(n+1)}^{\prime}-t_{p n}^{\prime} \\
& u_{p n}^{\prime}=\frac{L_{n}}{\Delta t_{p n}^{\prime}} \quad(n=1 \sim 5)
\end{aligned}
$$

where $t_{c n}^{\prime}(\mathrm{s})$ is the elapsed time of the solute centroid travel from the solute injection point to the $n$th sensor, which is derived from the fitted curves; $\Delta t_{c n}^{\prime}(\mathrm{s})$ is the elapsed time of the solute centroid travel between adjacent sensors; $u_{c n}^{\prime}$ $\left(\mathrm{m} \mathrm{s}^{-1}\right)$ is the average velocity of centroid travel between adjacent sensors obtained by the new and improved method; $t_{p n}^{\prime}(\mathrm{s})$ is the elapsed time of peak concentration travel from the solute injection point to the $n$th sensor, which is derived from the fitted curves; $\Delta t_{p n}^{\prime}$ (s) is the duration of peak concentration travels between adjacent sensors; and $u_{p n}^{\prime}\left(\mathrm{m} \mathrm{s}^{-1}\right)$ is the average velocity of peak concentration travel between adjacent sensors obtained by the new and improved method.

The solution of Pulse Boundary Model effectively fit the measured data and obtains the correct $t_{p}^{\prime}$ and centroid time $t_{c}^{\prime}$. Then, by calculating the velocities between two adjacent sensors, but not those between the salt injection positions to the measured sensors, can reduce the velocities measured at short distances and determine the newly calculated centroid velocity $u_{c n}^{\prime}$ and peak concentration velocity $u_{p n}^{\prime}$. On this basis, this method is considered suitable for the cases with shallow flow in $\mathrm{cm}$ for application in the field.

\section{METHODS AND MATERIALS}

An experimental flume that is $4 \mathrm{~m}$ long and $15 \mathrm{~cm}$ wide was used to simulate the water flow wherein the solute was transported. Figure 3 shows the experimental system. The system included a computer installed with specially designed software for controlling the salt solute injection and sensed data logging, an interface unit, electric conductivity sensors, a salt solute injector, the flume, and the water supply. The experiments involved a combination of three flow rates $\left(\mathrm{Q}=12,24\right.$, and $\left.48 \mathrm{~L} \mathrm{~min}^{-1}\right)$ and three slope gradients $\left(\mathrm{S}=4,8\right.$, and $\left.12^{\circ}\right)$. Regulated water flow was introduced into the flume from the upper end. Once the flow stabilized (within $1 \mathrm{~min}$ fluctuation), approximately $6 \mathrm{ml}$ of highly saturated salt solution $(\mathrm{KCl})$ was injected at a location $1 \mathrm{~m}$ from the upper end of the flume, allowing a certain distance to achieve a steady flow. The salt solution was injected into the water flow using a computer-controlled electrical valve. The 6 sensors were located at 5, 30,60,90,120, and $150 \mathrm{~cm}$ from the solute injector. The electrical conductivity values measured at these six locations were logged into the computer through the specially designed data logger, controlled by the specially designed software.

The experimentally obtained data were fit into Eq. (6), then the fitted curves, $u_{e}$, and $t_{p}^{\prime}$ were calculated. The centroid time $t_{c}^{\prime}$ were obtained from the fitted curves with Eq. (3), then the centroid and peak concentration velocities were determined by the new and improved method.

\section{RESULTS AND DISCUSSION}

\subsection{Time Calculation}

Figure 4 shows the solute transport progress as the experimental data (dotted curves) and the trends fitted with the analytic solution function (smooth curves) as given by the solution of Pulse Boundary Model [Eq. (6)] along slopes. As shown in Fig. 4 and Table 1, although the overall trend of the curve is clear, the measured data had varying fluctuations. Obtaining a reasonable centroid time and peak concentration time $\left(t_{p}\right)$ is difficult with the traditional salt tracer method. Thus, the calculated centroid velocities varied dramatically, such as from -0.44 to $11.84 \mathrm{~m} \mathrm{~s}^{-1}$ under flow rate of $48 \mathrm{~L} \mathrm{~min}^{-1}$ and slope gradients of $4^{\circ}$. Furthermore, accurate manual identification of peak time is difficult because the water flow is not an identical laminar flow or salt is adsorbed by the sediment.

The coefficients of determination calculated at all positions and for all experimental treatments were $0.92-0.99$. No significant differences were found for data sets obtained at different measurement positions, for different slope gradients, and under different flow rates. The results indicated that the model effectively fitted the solute transport processes (Fig. 4). The fitted curves followed the peak and the fluctuations of the experimentally obtained curves, from which we can derive the only sure values of peak concentration time $\left(t_{p}^{\prime}\right)$ and reasonable centroid time $\left(t_{c}^{\prime}\right)$. Figure 5 shows the comparison of centroid times calculated by traditional salt tracer method $\left(t_{c}\right)$ and the new and improved method $\left(t_{c}^{\prime}\right)$. The centroid times obtained using the traditional salt tracer method was higher than those from the new and improved method. The possible reason is the salt sorption by the sediment of the slope, which delayed the solute transportation. On the one hand, the pulse tracer curve extended to the right and exhibited several minor wave crests ( $\mathrm{Li}$ et al. 1996). On the other hand, the varying amounts of salt sorption by the different slope surfaces caused the different shape and volatility of the measurement curve, which caused different results of adjacent sensors. The centroid times calculated by the traditional salt tracer method showed greater variations than those obtained by the new and improved method. According to the assumption and experiment setting of this 
study, the velocity measured by different sensors should be consistent in a certain experiment. Thus, the calculated results of centroid time by the new and improved method was more reasonable.

Figure 6 shows the difference between $t_{c}$ and $t_{p}$, which generally indicated that $t_{p}<t_{c}$. This result is consistent with the analysis by Taylor (1954) that the tracer concentration distributed with time was right-skewed for the finite travel distance. The reason associated with the dispersion of the solute. Figure 6 also indicated that the difference between $t_{c}$ and $t_{p}$ decreased with the increase of the slope gradients. Furthermore, we can conclude from Eqs. (1) and (2) that $u_{p}>u_{c}$.

\subsection{Results of Velocity Measurement}

Figure 7 shows the velocities computed by the new and improved method, traditional salt tracer method, and Pulse Boundary Model under different conditions. As mentioned above, time calculated results by the traditional salt tracer method were not reasonable, and thus we used those from the fitted curves to calculate the velocity using the traditional salt tracer method [Eqs. (1) - (3)].

The results showed that peak concentration velocity was a little higher than centroid velocity. The difference between centroid velocities obtained by the traditional salt tracer method and the Pulse Boundary Model at different distances from the $\mathrm{KCl}$ solution injection position was not significant, which confirmed the theoretical analysis from Eqs. (7) to (15). Furthermore, the results indicated that the traditional salt tracer method obtained low velocities at short distances from the solute injection, similar to the Pulse Boundary Model method.

Centroid and peak concentration velocities obtained by the new and improved method were not significantly different. Both were higher than those of the traditional ones and of the Pulse Boundary Model. The differences increase with slope gradients and flow rate, which indicated that the Pulse Boundary Model resulted in greater errors at higher flow rate and steeper slope gradients. Figure 7 also shows that velocity by traditional salt tracer method increased with distances from the $\mathrm{KCl}$ solution injection point while that

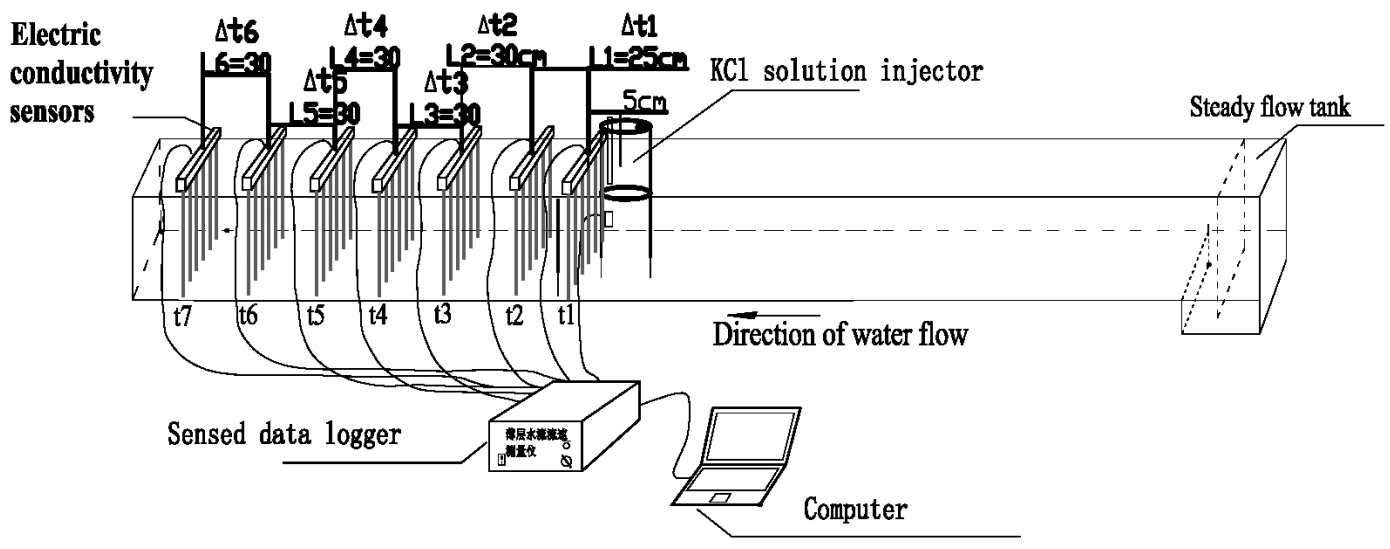

Fig. 3. Sketch of the experimental equipment system.

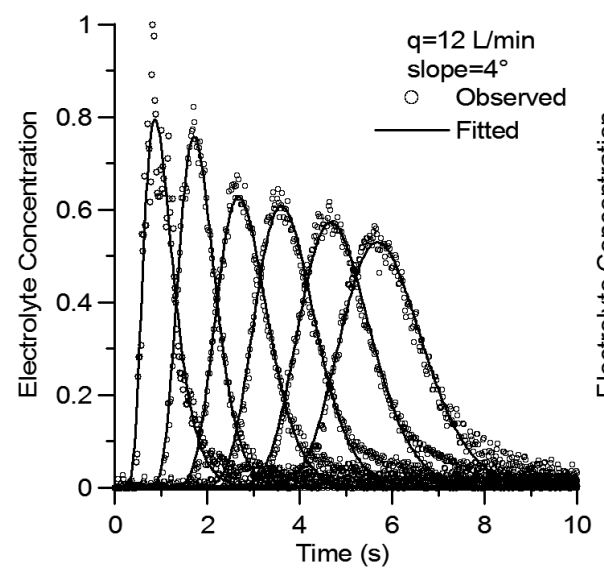

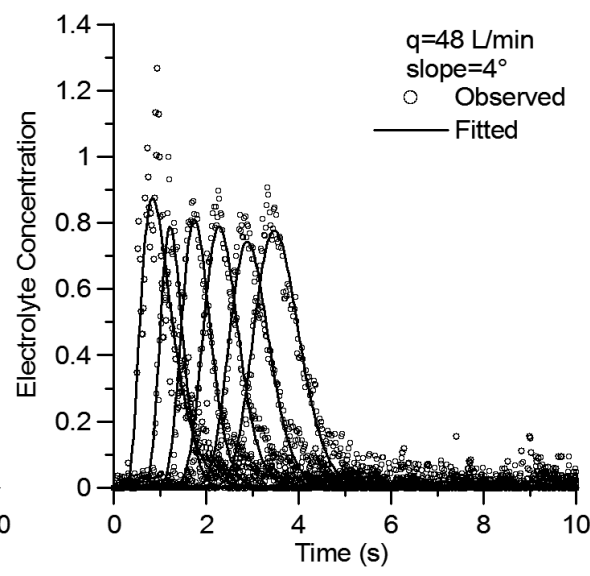

Fig. 4. Solute transport progress. 
Table 1 . Time and centroid velocity calculation results by traditional salt tracer method.

\begin{tabular}{|c|c|c|c|c|c|c|c|c|c|}
\hline & \multicolumn{3}{|c|}{$4^{\circ}$} & \multicolumn{3}{|c|}{$8^{\circ}$} & \multicolumn{3}{|c|}{$12^{\circ}$} \\
\hline & $x(\mathbf{m})$ & $t_{c}(\mathbf{s})$ & $u_{c}\left(\mathrm{~m} \mathrm{~s}^{-1}\right)$ & $x(\mathbf{m})$ & $t_{c}(\mathbf{s})$ & $u_{c}\left(\mathrm{~m} \mathrm{~s}^{-1}\right)$ & $x(\mathbf{m})$ & $t_{c}(\mathrm{~s})$ & $u_{c}\left(\mathrm{~m} \mathrm{~s}^{-1}\right)$ \\
\hline \multirow{6}{*}{$12 \mathrm{~L} \mathrm{~min}^{-1}$} & 0.05 & 1.98 & 0.03 & 0.05 & 1.55 & 0.03 & 0.05 & 1.62 & 0.03 \\
\hline & 0.3 & 2.09 & 2.14 & 0.3 & 2.69 & 0.22 & 0.3 & 3.22 & 0.16 \\
\hline & 0.6 & 3.26 & 0.26 & 0.6 & 3.52 & 0.36 & 0.6 & 3.30 & 4.04 \\
\hline & 0.9 & 4.18 & 0.33 & 0.9 & 3.91 & 0.77 & 0.9 & 3.55 & 1.20 \\
\hline & 1.2 & 5.16 & 0.31 & 1.2 & 4.81 & 0.33 & 1.2 & 4.12 & 0.52 \\
\hline & 1.5 & 6.04 & 0.34 & 1.5 & 5.37 & 0.54 & 1.5 & 4.83 & 0.43 \\
\hline \multirow{6}{*}{$24 \mathrm{~L} \mathrm{~min}^{-1}$} & 0.05 & 1.11 & 0.05 & 0.05 & 1.24 & 0.04 & 0.05 & 2.92 & 0.02 \\
\hline & 0.3 & 1.54 & 0.58 & 0.3 & 3.42 & 0.11 & 0.3 & 1.76 & -0.21 \\
\hline & 0.6 & 2.61 & 0.28 & 0.6 & 2.21 & -0.25 & 0.6 & 2.53 & 0.39 \\
\hline & 0.9 & 3.05 & 0.69 & 0.9 & 2.87 & 0.45 & 0.9 & 2.84 & 0.95 \\
\hline & 1.2 & 3.78 & 0.41 & 1.2 & 3.43 & 0.54 & 1.2 & 3.28 & 0.69 \\
\hline & 1.5 & 4.47 & 0.43 & 1.5 & 4.48 & 0.29 & 1.5 & 4.33 & 0.28 \\
\hline \multirow{6}{*}{$48 \mathrm{~L} \mathrm{~min}^{-1}$} & 0.05 & 2.58 & 0.02 & 0.05 & 1.84 & 0.03 & 0.05 & 0.87 & 0.06 \\
\hline & 0.3 & 2.91 & 0.76 & 0.3 & 1.97 & 1.90 & 0.3 & 1.37 & 0.49 \\
\hline & 0.6 & 2.23 & -0.44 & 0.6 & 2.31 & 0.87 & 0.6 & 2.33 & 0.31 \\
\hline & 0.9 & 3.39 & 0.26 & 0.9 & 2.54 & 1.35 & 0.9 & 2.08 & -1.20 \\
\hline & 1.2 & 3.42 & 11.84 & 1.2 & 3.48 & 0.32 & 1.2 & 3.21 & 0.27 \\
\hline & 1.5 & 4.47 & 0.28 & 1.5 & 3.75 & 1.10 & 1.5 & 3.04 & -1.72 \\
\hline
\end{tabular}
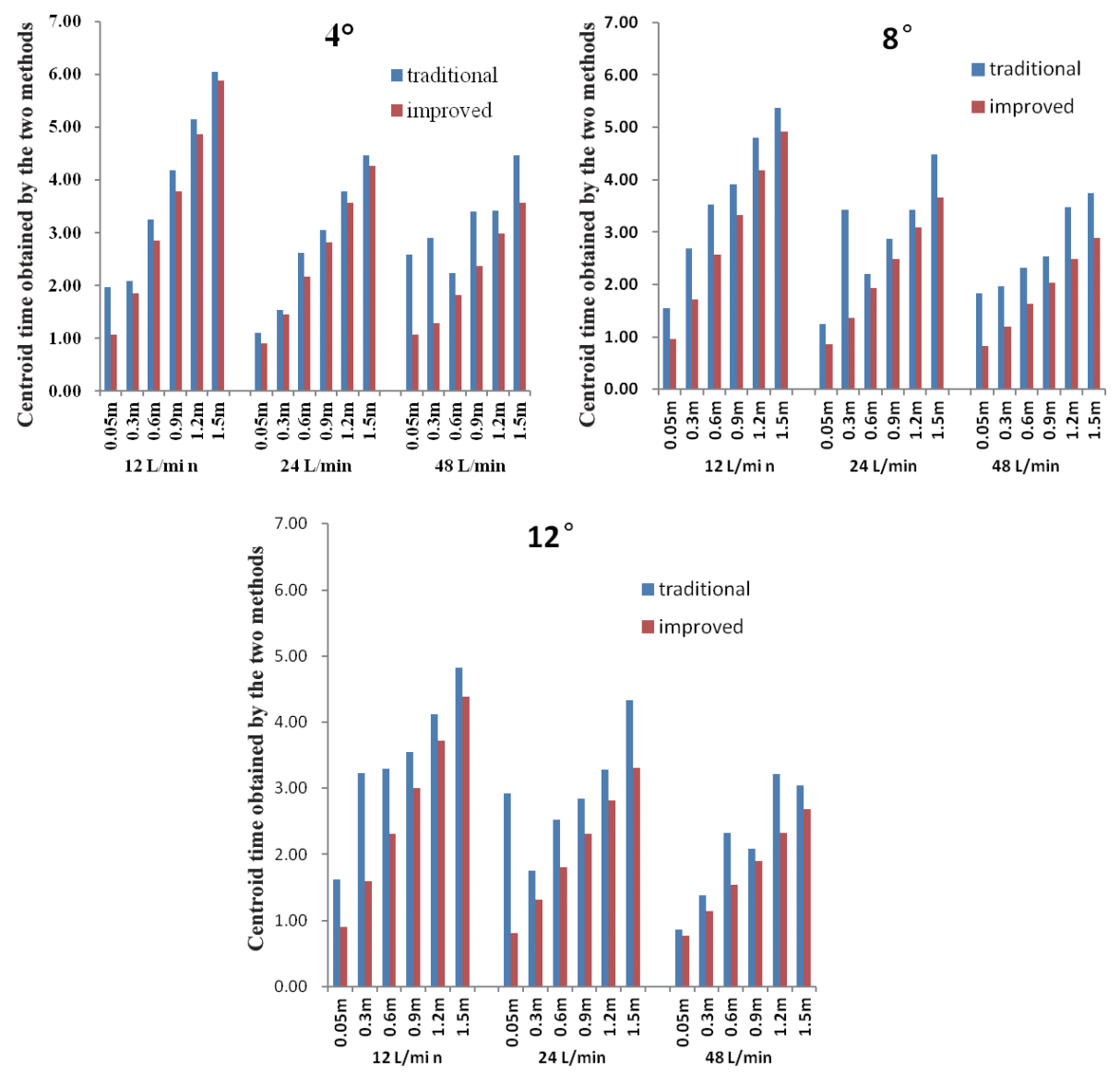

Fig. 5. Comparison of centroid time calculated by traditional and the improved salt tracer methods. 


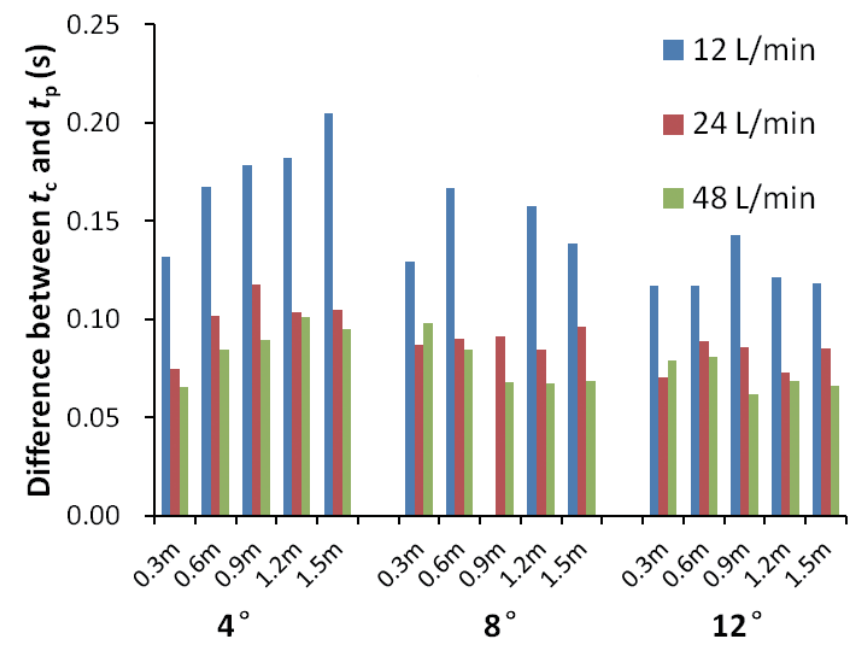

Fig. 6. Difference between $t_{\mathrm{c}}$ and $t_{\mathrm{p}}$ under different conditions.
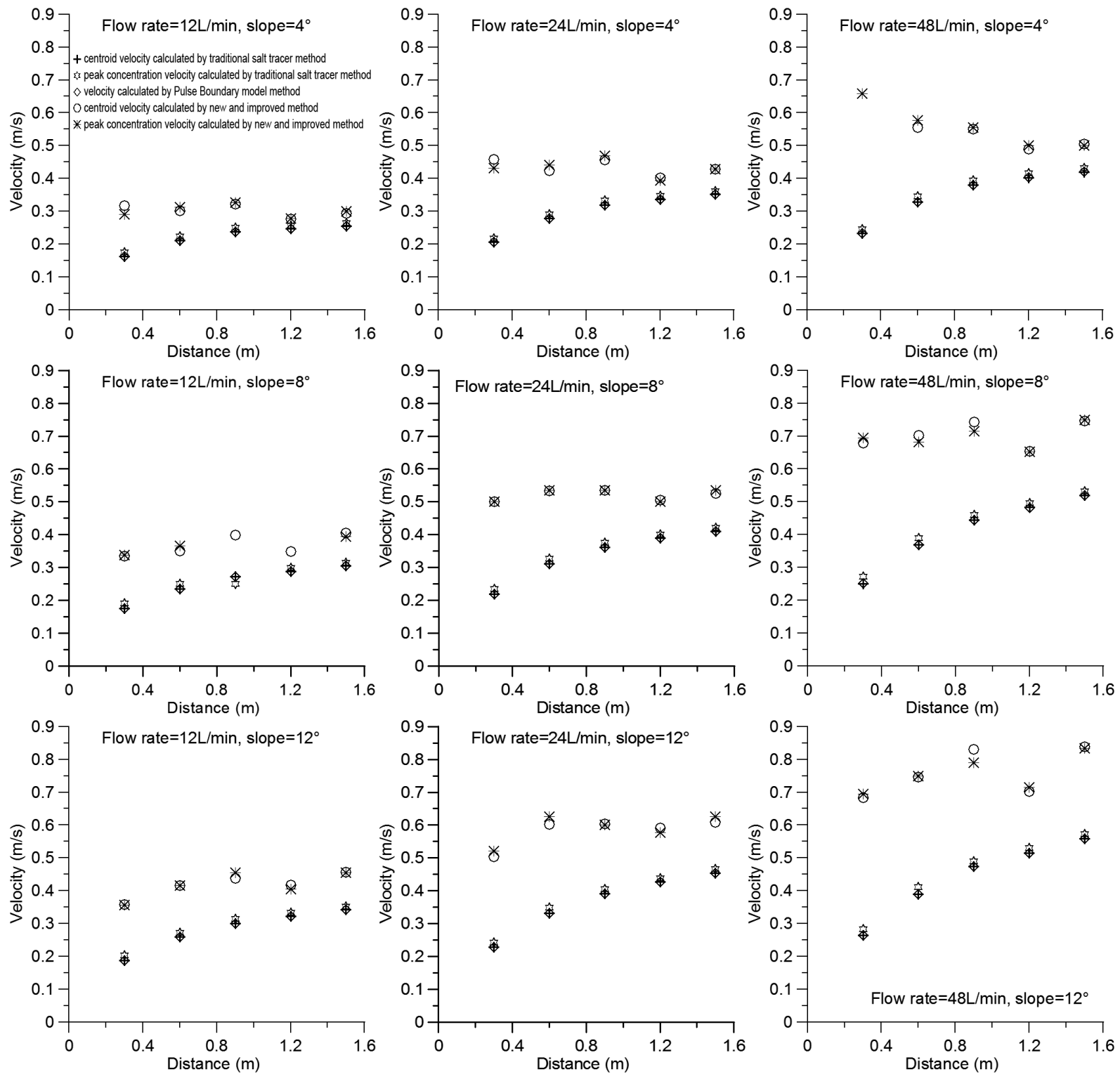

Fig. 7. Velocity calculated by the improved and traditional salt tracer methods and Pulse Boundary Model. 
of the new and improved method varied very little along the slope, which indicated an almost steady water flow. The measured velocities increased with distance from the solute injector and stablized after $60 \mathrm{~cm}$ for the flume slope of $12^{\circ}$. The possible reason is that the water flow is still accelerating in the upper part of the flume. In other words, velocities obtained by the new and improved method showed no increasing trend along the slope such as that calculated by the traditional salt tracer method. Thus, the new and improved method can effectively eliminate the error at short distances from the solute injection.

\subsection{Comparison of the New and Improved Method and Volumetric Method}

For comparison purposes, volumetric method was also used to verify the validity of the new and improved method, and the measurement precision was quantitatively shown under every experimental condition (Table 2 and Fig. 8). The volumetric method used a traditional needle approach to determine the flow depth, with which the average velocity was determined by dividing the flow rate by the product of the flume width and depth.

$u_{v}=\frac{Q}{w d}$

where $Q\left(\mathrm{~m}^{3} \mathrm{~s}^{-1}\right)$ is the flow rate; $w(\mathrm{~m})$ is the flume width; $d(\mathrm{~m})$ is the flume depth. The velocities by these different methods under varying experimental conditions were linear fitted. Figure 8 shows the regressed results, with the confidence interval of $95 \%$. The error was calculated by:

$e=\left(\frac{u_{v}-u_{n}}{u_{n}}\right) \times 100 \%$

Table 2. Error of the new and improved method relative to volumetric method.

\begin{tabular}{|c|c|c|c|c|c|c|}
\hline \multirow{2}{*}{ Slope $\left({ }^{\circ}\right)$} & \multirow{2}{*}{ Flow rate $\left(\mathrm{L}\right.$ min $\left.^{-1}\right)$} & \multirow{2}{*}{ Velocity by volumetric method $u_{v}\left(\mathrm{~m} \mathrm{~s}^{-1}\right)$} & \multicolumn{2}{|c|}{ Velocity by the new and improved method $\left(\mathrm{m} \mathrm{s}^{-1}\right)$} & \multicolumn{2}{|c|}{ Error $(\%)$} \\
\hline & & & uc' & up' & $\mathbf{u c}^{\prime}$ & up' \\
\hline \multirow{3}{*}{4} & 12 & 0.29 & 0.3 & 0.3 & 3.33 & 3.33 \\
\hline & 24 & 0.34 & 0.43 & 0.43 & 20.93 & 20.93 \\
\hline & 48 & 0.42 & 0.53 & 0.56 & 20.75 & 25.00 \\
\hline \multirow{3}{*}{8} & 12 & 0.33 & 0.37 & 0.41 & 10.81 & 19.51 \\
\hline & 24 & 0.41 & 0.52 & 0.52 & 21.15 & 21.15 \\
\hline & 48 & 0.55 & 0.71 & 0.7 & 22.54 & 21.43 \\
\hline \multirow{3}{*}{12} & 12 & 0.38 & 0.42 & 0.42 & 9.52 & 9.52 \\
\hline & 24 & 0.42 & 0.58 & 0.59 & 27.59 & 28.81 \\
\hline & 48 & 0.54 & 0.76 & 0.76 & 28.95 & 28.95 \\
\hline
\end{tabular}

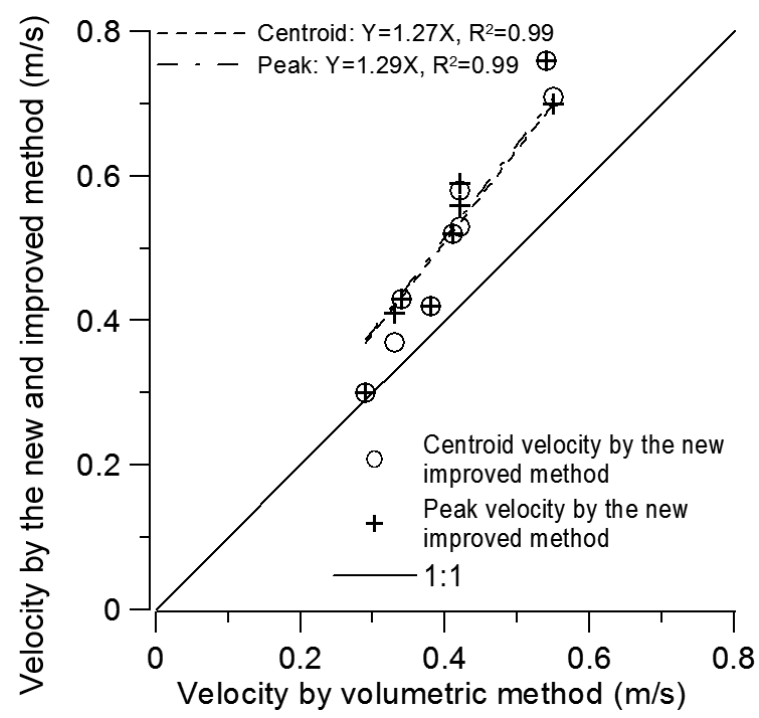

Fig. 8. Comparison of the velocities calculated by the new and improved and volumetric methods. 
where $u_{n}$ is the centroid or peak velocity of the new and improved method and $u_{v}$ is volumetric velocity.

Figure 8 and Table 2 shows that the velocities measured by the volumetric method were 0.79 (centroid velocity, $R^{2}=0.99$ ) and 0.78 (peak velocity, $R^{2}=0.99$ ) times of those estimated by the new and improved method. The multiple relationships between velocities of the two methods were close to that between the volumetric method and Measured Boundary Condition model, which was 0.79 (Lei et al. 2010). All the data points lay on the right side of the line of 1:1, which indicates that all velocities of the new and improved method were higher than those estimated by volumetric velocity, with the error of $3.33-28.95 \%$. The error of both the centroid and peak velocities increase with the slope and gradients, which indicates a velocity increase. The possible reason is the visual error of the flow depth measurement. The flow depth was typically very shallow, ranging $5-13 \mathrm{~mm}$ for the flow rates and slope gradients used in these experiments. The inertial and aqueous tension effect, water measured sensor, vernier caliper with a certain width of $2 \mathrm{~mm}$ made a wave at the fore-side from the flow direction, which indicates an error of $15-40 \%$ in measuring the flow depth. This error increased with the increase of flow velocity, slope gradients, and flow rate, which caused greater turbulence intensity. As the flow velocity increased, the flow turbulence and the error of depth velocity measurement also increased.

The peak concentration velocities were 0.999 times those of centroid velocities obtained by the new and improved method $\left(R^{2}=0.999\right)$, which confirmed that the centroid and peak concentration velocities were almost identical. With the new and improved method, either the centroid or peak times can be used to obtain higher velocity accuracy. Considering all these factors, we can conclude that the new and improved method can calculate shallow flow velocity at short distances from the solute injection without the common error obtained from traditional methods.

\subsection{Relative Errors at Different Positions of the Pulse Boundary Model Method}

Figure 7 shows that the measured velocities increased with the distance from the solute injection point. This finding is unlike that of Lei et al. (2013) who measured the water flow velocity within a gravel layer using the Pulse Boundary Model and found that the measured velocities increased with the distance from the solute injection point but stabilized after a distance of $60 \mathrm{~cm}$. In the present study, the measured velocities increased with the distance between the solute injector and measurement location under different flow rates and slope gradients, the increase rate decreased gradually with the distance. However, velocities did not stabilize even at the longest distance $(1.5 \mathrm{~m})$ except for the gentler slope $\left(4^{\circ}\right)$.
The new and improved method obtained consistent centroid and peak concentration velocities. As such, taking the average centroid velocity ( $\overline{u_{c}^{\prime}}$ shown in Table 2$)$ of the five locations $(0.3,0.6,0.9,1.2,1.5 \mathrm{~m})$ as the real velocity, we can estimate the relative errors in measured flow velocities using Pulse Boundary Model under different flow rates and slope gradients at different locations (Table 2). The relative errors at different locations from the solute injector ranged $15-65 \%$ (Table 3), which were much higher than the relative errors of velocity measured within a gravel layer by the Pulse Boundary Model (with the highest of 20\%).

\section{CONCLUSION}

The Pulse Boundary Model is an efficient method for velocity measurement. However, because of inappropriate assumption of pulse boundary conditions, this method produces low velocity. To reduce such errors, we present an improved approach by combining the Pulse Boundary Model and traditional solute tracer method and calculated the velocities of every two adjacent sensors. The results showed that the peak velocities were 0.999 times of the centroid velocities. In addition, the velocities estimated by the new method agreed well with those measured by the volumetric method, which were 0.79 (centroid velocity) and 0.78 (peak velocity) times of those by the new method. The centroid velocities calculated by the traditional salt tracer method and by Pulse Boundary Model method at different distances from the $\mathrm{KCl}$ solution injection position were not significantly different. However, they were significantly lower than those by the new and improved method, which obtained significantly greater measurement accuracy not only at short distance but along the entire slope. Furthermore, unlike the traditional salt tracer method, the centroid and peak concentration velocities by the new and improved method were almost identical. As such, with the new improved method, either centroid or peak time to obtain velocity with greater precision.

The relative errors of the velocities obtained by Pulse Boundary Model at different locations from the solute injector ranged $15-65 \%$, which increased with flow rate. These errors can be reduced by using the new and improved method and thereby increase the accuracy in measuring water flow velocity. The new improved method provides a precise and efficient approach in calculating shallow velocity at short distances.

Acknowledgements This work was supported by the Natural Science Foundation of China under Project No. 41501302, Agricultural water transportation based on multi-process driving mechanism for improving water use efficiency (No. 51321001), and China Postdoctoral Science Foundation funded project under Project No. 2015M570182. 
Table 3. The relative error at different distance/position of the Pulse Boundary Model method.

\begin{tabular}{|c|c|c|c|c|c|c|c|}
\hline & \multirow{2}{*}{$x(\mathbf{m})$} & \multicolumn{2}{|c|}{$12 \mathrm{~L} \mathrm{~min}^{-1}$} & \multicolumn{2}{|c|}{$24 \mathrm{~L} \mathrm{~min}^{-1}$} & \multicolumn{2}{|c|}{$48 \mathrm{~L} \mathrm{~min}^{-1}$} \\
\hline & & $E_{\mathrm{c}}(\%)$ & $\overline{u_{c}^{\prime}}\left(\mathrm{m} \mathrm{s}^{-1}\right)$ & $E_{\mathrm{c}}(\%)$ & $\overline{u_{c}^{\prime}}\left(\mathrm{m} \mathrm{s}^{-1}\right)$ & $E_{\mathrm{c}}(\%)$ & $\overline{u_{c}^{\prime}}\left(\mathrm{m} \mathrm{s}^{-1}\right)$ \\
\hline \multirow{5}{*}{$4^{\circ}$} & 0.3 & 45.99 & \multirow{5}{*}{0.30} & 52.04 & \multirow{5}{*}{0.37} & & \multirow{5}{*}{0.42} \\
\hline & 0.6 & 29.76 & & 35.44 & & 56.04 & \\
\hline & 0.9 & 20.60 & & 25.73 & & 37.92 & \\
\hline & 1.2 & 17.73 & & 21.69 & & 28.30 & \\
\hline & 1.5 & 15.05 & & 18.21 & & 23.96 & \\
\hline \multirow{5}{*}{$8^{\circ}$} & 0.3 & 52.56 & \multirow{5}{*}{0.43} & 57.88 & \multirow{5}{*}{0.52} & 64.65 & \multirow{5}{*}{0.58} \\
\hline & 0.6 & 36.81 & & 40.19 & & 48.03 & \\
\hline & 0.9 & 26.69 & & 30.58 & & 37.46 & \\
\hline & 1.2 & 22.36 & & 25.19 & & 32.11 & \\
\hline & 1.5 & 17.58 & & 21.15 & & 26.90 & \\
\hline \multirow{5}{*}{$12^{\circ}$} & 0.3 & 55.28 & \multirow{5}{*}{0.53} & 60.52 & \multirow{5}{*}{0.71} & 65.34 & \multirow{5}{*}{0.76} \\
\hline & 0.6 & 38.35 & & 42.81 & & 48.76 & \\
\hline & 0.9 & 28.64 & & 32.70 & & 37.73 & \\
\hline & 1.2 & 23.22 & & 26.44 & & 32.19 & \\
\hline & 1.5 & 18.43 & & 21.76 & & 26.52 & \\
\hline
\end{tabular}

\section{REFERENCES}

Abrantes, J. R. C. B., R. B. Moruzzi, A. Silveira, and J. L. M. P. de Lima, 2018: Comparison of thermal, salt and dye tracing to estimate shallow flow velocities: Novel triple-tracer approach. J. Hydrol., 557, 362-377, doi: 10.1016/j.jhydrol.2017.12.048. [Link]

Bresler, E., 1973: Simultaneous transport of solutes and water under transient unsaturated flow conditions. Water Resour. Res., 9, 975-986, doi: 10.1029/ WR009i004p00975. [Link]

Bruun, H. H., 1996: Hot-wire anemometry: Principles and signal analysis. Meas. Sci.Tech., 7, doi: 10.1088/09570233/7/10/024. [Link]

Chang, N.-B., A. James Crawford, G. Mohiuddin, and J. Kaplan, 2015: Low flow regime measurements with an automatic pulse tracer velocimeter (APTV) in heterogeneous aquatic environments. Flow Meas. Instrum., 42, 98-112, doi: 10.1016/j.flowmeasinst.2014.12.010. [Link]

Dunkerley, D., 2001: Estimating the mean speed of laminar overland flow using dye injection-uncertainty on rough surfaces. Earth Surf. Process. Landf., 26, 363-374, doi: 10.1002/esp.185. [Link]

Elder, J. W., 1959: The dispersion of marked fluid in turbulent shear flow. J. Fluid Mech., 5, 544-560, doi: 10.1017/S0022112059000374. [Link]

Emmett, W. W., 1970: The hydraulics of overland flow on hillslopes. Professional Paper 662- A, U.S. Government Printing Office, Washington, D.C., doi: 10.3133/
pp662A. [Link]

Giménez, R., O. Planchon, N. Silvera, and G. Govers, 2004: Longitudinal velocity patterns and bed morphology interaction in a rill. Earth Surf. Process. Landf., 29, 105114, doi: 10.1002/esp.1021. [Link]

Horton, R. E., H. R. Leach, and R. Van Vliet, 1934: Laminar sheet-flow. Eos, Trans. AGU, 15, 393-404, doi: 10.1029/TR015i002p00393. [Link]

Hyun, B. S., R. Balachandar, K. Yu, and V. C. Patel, 2003: Assessment of PIV to measure mean velocity and turbulence in open-channel flow. Exp. Fluid., 35, 262267, doi: 10.1007/s00348-003-0652-7. [Link]

Lei, T., W. Xia, J. Zhao, Z. Liu, and Q. Zhang, 2005: Method for measuring velocity of shallow water flow for soil erosion with an electrolyte tracer. J. Hydrol., 301, 139-145, doi: 10.1016/j.jhydrol.2004.06.025. [Link]

Lei, T., R. Chuo, J. Zhao, X. Shi, and L. Liu, 2010: An improved method for shallow water flow velocity measurement with practical electrolyte inputs. J. Hydrol., 390, 45-56, doi: 10.1016/j.jhydrol.2010.06.029. [Link]

Lei, T., Y. Yan, X. Shi, R. Chuo, and J. Zhao, 2013: Measuring velocity of water flow within a gravel layer using an electrolyte tracer method with a Pulse Boundary Model. J. Hydrol., 500, 37-44, doi: 10.1016/j.jhydrol.2013.07.025. [Link]

Li, G., A. D. Abrahams, and J. F. Atkinson, 1996: Correction factors in the determination of mean velocity of overland flow. Earth Surf. Process.Landf., 21, 509-515, doi: 10.1002/(SICI)1096-9837(199606)21:6<509::AIDESP613>3.0.CO;2-Z. [Link] 
Li, Y., M. Yuan, and C. Li, 1997: Hot film technique in the application of flow velocity measurement. Experiments and Measurements in Fluid Mechanics, 11, 4550. (in Chinese)

Liu, P., X. Li, and W. Wang, 2007: Runoff flow velocity measurement system using photoelectric sensor and tracing method. Transactions of the CSAE, 23, 116120. (in Chinese)

Liu, P., X. Li, W. Wang, T. Lei, and J. Zhang, 2008: Runoff flow velocity measurement system based on correlation method. Transactions of the CSAE, 24, 48-52. (in Chinese)

Luk, S. H. and W. Merz, 1992: Use of the salt tracing technique to determine the velocity of overland-flow. Soil Tech., 5, 289-301.

Myers, T. G., 2002: Modeling laminar sheet flow over rough surfaces. Water Resour. Res., 38, 12-1-12-12, doi: 10.1029/2000WR000154. [Link]

Pan, C. and Z. Shangguan, 2006: Runoff hydraulic characteristics and sediment generation in sloped grassplots under simulated rainfall conditions. J. Hydrol., 331, 178-185, doi: 10.1016/j.jhydrol.2006.05.011. [Link]

Planchon, O., N. Silvera, R. Gimenez, D. Favis-Mortlock, J. Wainwright, Y. L. Bissonnais, and G. Govers, 2005: An automated salt-tracing gauge for flow-velocity measurement. Earth Surf. Process. Landf., 30, 833-
844, doi: 10.1002/esp.1194. [Link]

Rouhipour, H, C. W. Rose, B. Yu, and H. Ghadiri, 1999: Roughness coefficients and velocity estimation in well-inundated sheet and rilled overland flow without strongly eroding bed forms. Earth Surf. Process. Landf., 24, 233-245, doi: 10.1002/(SICI)10969837(199903)24:3<233::AID-ESP949>3.0.CO;2-T. [Link]

Shi, X., F. Zhang, T. Lei, R. Chuo, S. Zhou, and Y. Yan, 2012: Measuring shallow water flow velocity with virtual boundary condition signal in the electrolyte tracer method. J. Hydrol., 452-453, 172-179, doi: 10.1016/j. jhydrol.2012.05.046. [Link]

Taylor, G. I., 1954: The dispersion of matter in turbulent flow through a pipe. Proc. Roy. Soc. Lond. Math. Phys. Sci., 223, 446-468, doi: 10.1098/rspa.1954.0130. [Link]

Xia, W., 2003: Study on velocity measurement of steady sheet flow with the electrolyte pulse method and its application. Northwest Sci-Tech Univerity of Agriculture and Forestry, Yangling, China.

Zhang, G., R. Luo, Y. Cao, R. Shen, and X. C. Zhang, 2010: Correction factor to dye-measured flow velocity under varying water and sediment discharges. J. Hydrol., 389, 205-213, doi: 10.1016/j.jhydrol.2010.05.050. [Link] 